\title{
A SHARP ESTIMATE IN AN OPERATOR INEQUALITY
}

\author{
R. MCEACHIN
}

(Communicated by Palle E. T. Jorgensen)

\begin{abstract}
Let $\mathscr{H}$ and $\mathscr{K}$ be Hilbert spaces, and suppose $A \in \mathscr{B}(\mathscr{H})$ and $B \in \mathscr{B}(\mathscr{K})$ are selfadjoint operators with $\operatorname{dist}(\sigma(A), \sigma(B)) \geq \delta>0$. It is known that for any $Q \in \mathscr{B}(\mathscr{K}, \mathscr{H})$ we must have $\frac{\pi}{2}\|A Q-Q B\| \geq \delta\|Q\|$. In this paper we give examples proving that $\pi / 2$ is sharp in this inequality.
\end{abstract}

\section{INTRODUCTION}

In 1983 Bhatia, Davis, and McIntosh (see [3]) proved the following

Theorem. Suppose $\mathscr{H}$ and $\mathscr{K}$ are Hilbert spaces, and let $A \in \mathscr{B}(\mathscr{H})$ and $B \in \mathscr{B}(\mathscr{K})$ be selfadjoint operators with $\operatorname{dist}(\sigma(A), \sigma(B)) \geq \delta>0$. Then there is some constant $c$ (independent of $A$ and $B$ ) such that $c|\|A Q-Q B|\|\geq \delta \mid\| Q\|\|$ for any $Q \in \mathscr{B}(\mathscr{K}, \mathscr{H})$ and any unitary invariant norm $\|\cdot\| \|$.

We will say a norm is unitary invariant, or $u i$, if $\||U T V|\|=|\|T \mid\|$ whenever $U$ and $V$ are unitary. Let $c$ be the smallest constant such that, with $A, Q$, and $B$ as above, the inequality

$$
c|\|A Q-Q B\|| \geq \delta\|\mid Q\| \|
$$

holds for arbitrary $u i$ norms. In [3] the authors establish $1.22<c<2$, and in [7] Sz.-Nagy proves $c \leq \pi / 2$. (This also follows from his earlier paper [6].) The purpose of this paper is to prove $c=\pi / 2$. To do this we give $n \times n$ matrices $A_{n}, Q_{n}$, and $B_{n}$ such that $A_{n}$ and $B_{n}$ are selfadjoint and $\delta\left\|Q_{n}\right\| /\left\|A_{n} Q_{n}-Q_{n} B_{n}\right\| \rightarrow \pi / 2$ as $n \rightarrow \infty$. Here $\|\cdot\|$ is the operator norm, and since this is unitary invariant it follows that $\pi / 2$ is sharp in $(*)$.

This inequality can be viewed as geometrical in nature. As seen from $[3,4]$, it is an important tool in the analysis of the spectral variation of selfadjoint or normal operators. It is well known that if a selfadjoint matrix $S$ undergoes a small selfadjoint perturbation $H$ then its eigenvalues cannot change very much. Results of this nature, for example, are surveyed in [1, Chapter 3]. Eigenvectors, however, can rotate through a large angle. For instance, two operators very close to $I$, and thus close to each other, could still have eigenspaces far apart. But suppose $\sigma(S)$ can be divided into two or more parts separated from each other.

Received by the editors November 12, 1990.

1980 Mathematics Subject Classification (1985 Revision). Primary 47A55, 47B15; Secondary $15 \mathrm{~A} 45$. 
Then for $T=S+H, \sigma(T)$ must have corresponding separated parts when $\||| H||$ is small, and a spectral subspace for one part of $\sigma(T)$ must be almost orthogonal to a spectral subspace for a different part of $\sigma(S)$. In this case, then, a small perturbation cannot rotate a spectral projection through a large angle. While bounds on the variation of $\sigma(S)$ are classical, bounds on the variation of spectral projections are newer and are obtained as a corollary to the $A Q-Q B$ inequality. Furthermore, this inequality and its corollaries can be generalized to yield important bounds on the variation of both the spectrum and the spectral subspaces of a normal operator. These results are chiefly developed in [3, 4], and the discussions in $[1$, Chapter $4 ; 2]$ explain their interconnections very well.

In the examples below, if $n$ is understood we let $\left(a_{i j}\right)$ denote the $n \times n$ matrix whose entry in the $i$ th row and $j$ th column is $a_{i j}$. The following discussion was first presented in my doctoral dissertation at the University of Illinois. At this time I wish to thank my advisor, Professor I. D. Berg, as well as Professor Chandler Davis at the University of Toronto for their assistance.

\section{The EXAMPLES}

For each $n$, let $A_{n}=\operatorname{diag}(2 n, 2 n-2,2 n-4, \ldots, 2)$ and let $B_{n}=$ $\operatorname{diag}(1,3,5, \ldots, 2 n-1)$. For $1 \leq i \leq n$, set $\lambda_{i}=(2 n-2 i+2) \in \sigma(S)$ and $\mu_{i}=(2 i-1) \in \sigma(T)$. Then $\delta=\operatorname{dist}(\sigma(A), \sigma(B))=1$, and for any $Q_{n}=\left(q_{i j}\right)$ we have $A_{n} Q_{n}-Q_{n} B_{n}=\left(\left(\lambda_{i}-\mu_{j}\right) q_{i j}\right)$. Now set $Q_{n}=\left(\left(\lambda_{i}-\mu_{j}\right)^{-2}\right)$, so that $C_{n}:=A_{n} Q_{n}-Q_{n} B_{n}=\left(\left(\lambda_{i}-\mu_{j}\right)^{-1}\right)=$

$$
\left[\begin{array}{ccccc}
\frac{1}{2 n-1} & \frac{1}{2 n-3} & \frac{1}{2 n-5} & \ldots & 1 \\
\frac{1}{2 n-3} & \frac{1}{2 n-5} & \frac{1}{2 n-7} & \ldots & -1 \\
\frac{1}{2 n-5} & \frac{1}{2 n-7} & \frac{1}{2 n-9} & \ldots & -\frac{1}{3} \\
\vdots & \vdots & \vdots & & \vdots \\
1 & -1 & -\frac{1}{3} & \ldots & \frac{-1}{2 n-3}
\end{array}\right]
$$

The following three propositions yield values for $\lim _{n}\left\|C_{n}\right\|$ and $\lim _{n}\left\|Q_{n}\right\|$.

Proposition 1. For $\mathbf{x}=(\ldots,-1 / 5,-1 / 3,-1,1,1 / 3,1 / 5, \ldots) \in \ell_{2}(Z)$ and $S$ the right shift $\mathbf{e}_{i} \mapsto \mathbf{e}_{i+1}$, we have $\left\langle S^{n} \mathbf{x}, \mathbf{x}\right\rangle=0$ for all $n \neq 0$.

Proof. It is sufficient to consider $n>0$. Let

$$
\begin{aligned}
\mathbf{x} & =\left(\ldots,-\frac{1}{3},-1,1, \frac{1}{3}, \ldots, \frac{1}{2 n-1}, \frac{1}{2 n+1}, \frac{1}{2 n+3}, \ldots\right) \\
S^{n} \mathbf{x} & =\left(\ldots, \frac{-1}{2 n+3}, \frac{-1}{2 n+1}, \frac{-1}{2 n-1}, \frac{-1}{2 n-3}, \ldots,-1,1, \frac{1}{3}, \ldots\right) .
\end{aligned}
$$

Note that there are $n$ positions where the entries have opposite signs and in all other positions the entries have the same sign. Then $\left\langle S^{n} \mathbf{x}, \mathbf{x}\right\rangle$ is the sum of $n$ negative terms and two (identical) series of positive terms. The negative terms 
contribute

$$
\begin{aligned}
\sum_{k=0}^{n-1} & \frac{1}{2 k+1} \cdot \frac{1}{2 k-(2 n-1)} \\
& =\frac{-1}{2 n} \sum_{k=0}^{n-1}\left[\frac{1}{2 k+1}-\frac{1}{2 k-(2 n-1)}\right] \\
& =\frac{-1}{2 n}\left[\sum_{k=0}^{n-1} \frac{1}{2 k+1}+\sum_{m=0}^{n-1} \frac{1}{2 m+1}\right] \quad(m=n-k+1) \\
& =\frac{-1}{n} \sum_{k=0}^{n-1} \frac{1}{2 k+1} .
\end{aligned}
$$

On the other hand, the positive terms sum to

$$
2 \sum_{k=0}^{\infty} \frac{1}{2 k+1} \cdot \frac{1}{2 n+2 k+1}=\frac{1}{n} \sum_{k=0}^{\infty}\left[\frac{1}{2 k+1}-\frac{1}{2 n+2 k+1}\right] .
$$

This is a telescoping sum after cancellation it reduces to $n^{-1} \sum_{k=0}^{n-1} 1 /(2 k+1)$, and so $\left\langle S^{n} \mathbf{x}, \mathbf{x}\right\rangle=0$ as desired.

Proposition 2. For $C_{n}$ as above, $\left\|C_{n}\right\| / \pi / 2$ as $n \rightarrow \infty$.

Proof. Consider the doubly infinite matrix

$$
T=\left[\begin{array}{ccccccc} 
& \vdots & \vdots & \vdots & \vdots & \vdots & \\
\cdots & 1 / 9 & 1 / 7 & 1 / 5 & 1 / 3 & 1 & \ldots \\
\cdots & 1 / 7 & 1 / 5 & 1 / 3 & 1 & -1 & \ldots \\
\cdots & 1 / 5 & 1 / 3 & 1 & -1 & -1 / 3 & \ldots \\
\cdots & 1 / 3 & 1 & -1 & -1 / 3 & -1 / 5 & \ldots \\
\cdots & 1 & -1 & -1 / 3 & -1 / 5 & -1 / 7 & \ldots \\
& \vdots & \vdots & \vdots & \vdots & \vdots &
\end{array}\right] .
$$

Each column has norm $\left(2\left(1+1 / 3^{2}+1 / 5^{2}+\cdots\right)\right)^{1 / 2}=\pi / 2$, and by Proposition 1 the columns are mutually orthogonal. Thus $T$ is a multiple of a unitary operator on $\ell_{2}(Z)$ and $\|T\|=\pi / 2$. Since $C_{n}$ is a submatrix of $T$ for any $n,\left\|C_{n}\right\| \leq \pi / 2$. Furthermore, $\left\|C_{n}\right\|$ increases because $C_{n}$ is a submatrix of $C_{n+1}$. By considering the lengths of the columns of $C_{n}$, it is immediate that $\left\|C_{n}\right\| \nearrow \pi / 2$ as $n \rightarrow \infty$.

Proposition 3. For $Q_{n}$ as above, $\left\|Q_{n}\right\| / \pi^{2} / 4$ as $n \rightarrow \infty$.

Proof. We know that $\frac{\pi}{2}\left\|C_{n}\right\| \geq\left\|Q_{n}\right\|$, and so by Proposition 2 we have $\pi^{2} / 4 \geq$ $\left\|Q_{n}\right\|$ for each $n$. Furthermore $\left\|Q_{n}\right\|$ increases because each $Q_{n}$ is a submatrix of $Q_{n+1}$. We will show that for any $\varepsilon>0$ there is some $n$ such that $\left\|Q_{n}\right\|>$ $\pi^{2} / 4-\varepsilon$. To this end define $s_{n}=2\left(1+1 / 3^{2}+1 / 5^{2}+\cdots+1 /(2 n-1)^{2}\right)$, and note that $\left\{s_{n}\right\} \nearrow \pi^{2} / 4$ as $n \rightarrow \infty$. For each $n$ let $\mathbf{x}$ be the $n$-vector $[1,1,1, \ldots, 1]^{T}$ and $\mathbf{y}=Q_{n} \mathbf{x}=$

$$
\left[\sum_{k=1}^{n} \frac{1}{(2 k-1)^{2}}, \sum_{k=1}^{n} \frac{1}{(2 k-3)^{2}}, \sum_{k=1}^{n} \frac{1}{(2 k-5)^{2}}, \ldots, \sum_{k=1}^{n} \frac{1}{(2 k-2 n+1)^{2}}\right]^{T} .
$$


If $y_{i}$ is the $i$ th component of $\mathbf{y}$ observe that $y_{2}, y_{n}>s_{1}$. Similarly, $y_{3}, y_{n-1}>$ $s_{2}, y_{4}, y_{n-2}>s_{3}$, and so on. If $\varepsilon>0$, choose $p$ large enough so that $s_{p}>\pi^{2} / 4-\varepsilon$. Then take $r$ so large that $\sqrt{r} / \sqrt{r+2 p+3}>s_{p} / s_{p+1}$ and set $n=r+2 p+3$. Then all coordinates of $\mathbf{y}$ except the first $p+2$ and the last $p+1$ are larger than $s_{p+1}$; that is, the $r$ components $y_{p+3}, y_{p+4}, \ldots, y_{p+r+2}$ are all greater than $s_{p+1}$, and so $\|\mathbf{y}\|>\sqrt{r} s_{p+1}$. Since $\|\mathbf{x}\|=\sqrt{n},\left\|Q_{n}\right\|>$ $\sqrt{r} s_{p+1} / \sqrt{n}=\sqrt{r} s_{p+1} / \sqrt{r+2 p+3}>s_{p}>\pi^{2} / 4-\varepsilon$. This establishes our proposition.

We now observe that since $\delta=1, \lim _{n}\left\|Q_{n}\right\|=\pi / 2$, and $\lim _{n}\left\|C_{n}\right\|=$ $\lim _{n}\left\|A_{n} Q_{n}-Q_{n} B_{n}\right\|=\pi^{2} / 4$, it follows that $\delta\left\|Q_{n}\right\| /\left\|A_{n} Q_{n}-Q_{n} B_{n}\right\| \rightarrow \pi / 2$ as $n \rightarrow \infty$. Therefore $c \geq \pi / 2$, where $c$ is the constant in $(*)$. Since $c \leq \pi / 2$ is known, the value $\pi / 2$ is sharp.

\section{CONCLUding REMARKS}

In this section we indicate an alternate derivation of $\lim _{n}\left\|C_{n}\right\|$ and $\lim _{n}\left\|Q_{n}\right\|$. First, note that $Q_{n}=C_{n} \circ C_{n}$, where $A \circ B$ denotes the Schur product of $A$ and $B$. (That is, if $A=\left(a_{i j}\right)$ and $B=\left(b_{i j}\right)$ then $A \circ B=\left(a_{i j} b_{i j}\right)$.) Then $\lim _{n}\left\|C_{n}\right\|$ and $\lim _{n}\left\|Q_{n}\right\|$ are easily deducible from the fact that, for $T$ as above, $\|T\|=\pi / 2$ and $\|T \circ T\|=\pi^{2} / 4$.

To prove $\|T\|=\pi / 2$, let $\mathscr{H}=L^{2}(-1 / 2,1 / 2)$, and define

$$
f(x)= \begin{cases}i & \text { if } x \in(-1 / 2,0], \\ -i, & \text { if } x \in(0,1 / 2) .\end{cases}
$$

Then let $T_{f}: \mathscr{H} \rightarrow \mathscr{H}$ be the linear map with $T_{f} h=f h$. If we choose the orthonormal basis $\{\exp (2 \pi i n x)\}_{n=-\infty}^{\infty}$ for $\mathscr{H}$, the matrix of $T_{f}$ in this basis is $M=\left(a_{i j}\right)_{i, j=-\infty}^{\infty}$, where

$$
a_{i j}= \begin{cases}0 & \text { if } i-j \text { is even }, \\ -2 / \pi(i-j) & \text { if } i-j \text { is odd }\end{cases}
$$

One can show that $\|T\|=\frac{\pi}{2}\|M\|$ and $\|M\|=\|f\|_{L^{\infty}}=1$. Therefore, $\|T\|=$ $\pi / 2$. The proof for $\|T \circ T\|$ is similar. Define

$$
g(x)= \begin{cases}4 x+1 & \text { if } x \in(-1 / 2,0] \\ -4 x+1 & \text { if } x \in[0,1 / 2)\end{cases}
$$

Then $T_{g}$ has matrix $M \circ M$, and in the same way as before, $\|T \circ T\|=$ $\frac{\pi^{2}}{4}\|M \circ M\|$ and $\|M \circ M\|=\|g\|_{L^{\infty}}=1$. Therefore $\|T \circ T\|=\pi^{2} / 4$.

With a little more work, one can now conclude that $\left\|C_{n}\right\| \rightarrow \pi / 2$ and $\left\|Q_{n}\right\| \rightarrow$ $\pi^{2} / 4$ as $n \rightarrow \infty$.

\section{REFERENCES}

1. R. Bhatia, Perturbation bounds for matrix eigenvalues, Pitman Research Notes in Math., no. 162, Longman, Essex, 1987.

2. R. Bhatia, Ch. Davis, and P. Koosis, An extremal problem in Fourier analysis with applications to operator theory, J. Funct. Anal. 82 (1989), 138-150.

3. R. Bhatia, Ch. Davis, and A. McIntosh, Perturbation of spectral subspaces and solution of linear operator equations, Linear Algebra Appl. 52-53 (1983), 45-67.

4. Ch. Davis and W. M. Kahan, The rotation of eigenvectors by a perturbation. III, SIAM J. Numer. Anal. 7 (1970), 1-46. 
5. R. McEachin, Analysis of an inequality concerning perturbation of self-adjoint operators, Doctoral disseration, Univ. of Illinois, Urbana, IL, October 1990.

6. B. Sz.-Nagy, Über die Ungleichung von H. Bohr, Math. Nachr. 9 (1953), 225-259.

7. B. Sz.-Nagy, Bohr inequality and an operator equation, Operators in indefinite metric spaces, scattering theory, and other topics (Bucharest, 1985), pp. 321-327; Oper. Theory: Adv. Appl., vol. 24, Birkhauser, Basel and Boston, MA, 1987.

Department of Mathematical Sciences, Indiana University-PuRdue University at Fort WAYNE, ForT WAYNE, INDIANA 46805

Current address: Department of Mathematics, University of the West Indies, Mona, Kingston 7, Jamaica 